\title{
Acute inflammation in horizontal incompletely impacted third molar with radiolucency in the elderly
}

This article was published in the following Dove Press journal:

Clinical Interventions in Aging

29 July 2009

Number of times this article has been viewed

\section{Minoru Yamaoka \\ Yusuke Ono \\ Masahiro Takahashi \\ Masahide Ishizuka \\ Takayuki Uchihashi \\ Kouichi Yasuda \\ Takashi Uematsu \\ Kiyofumi Furusawa}

Matsumoto Dental University, Oral and Maxillofacial Surgery, Shiojiri, Nagano, Japan
Correspondence: Minoru Yamaoka, Oral and Maxillofacial Surgery, Matsumoto Dental University, Shiojiri, Nagano 399-078I Japan Fax +8I 263 5I 2076 Email yamaoka@po.mdu.ac.jp
Abstract: Although radiolucency has been shown as a risk of infection, the poorly understood effects of aging on radiolucency correlate with acute pericoronitis, which has a high risk of infection extending any complications. We reviewed the records of 346 consecutive patients aged more than 41 years to evaluate whether pericoronal radiolucency below the crown in mandibular horizontal incompletely impacted third molars is related to acute inflammation. The frequency of acute inflammation in teeth with pericoronal radiolucency below the crown was similar to that in teeth without; however, the odds ratio of acute inflammation exhibited in women aged more than 61 years compared to women aged $41-50$ years was 9.77 (95\% confidence interval [CI]: $1.67-57.29 ; P<<0.05)$, and in women aged more than 61 years compared to women aged 51-60 years was 26.25 (95\% CI: 2.94-234.38; $P<0.01$ ). The odds ratio of severe acute inflammation exhibited in men aged more than 61 years compared to men aged $41-50$ years was 16.67 (95\% CI: $1.76-158.27 ; P<0.01)$. These odds ratios indicate an association of acute pericoronitis, including the severe forms of acute inflammation that result from pericoronitis, with pericoronal radiolucency below the crown in the elderly.

Keywords: radiolucency, mandible, third molar, acute inflammation, aging

\section{Introduction}

A close relationship between radiolucency and periapical or periodontal inflammatory lesion has been universally shown. A structure that appears radiolucent on a radiograph lacks density ${ }^{1}$ or indicates bone destruction, ${ }^{2,3}$ and permits the passage of the X-ray with little or no resistance. ${ }^{1}$ The terms "radiolucent" and "radiopaque" are relative depending on the density and thickness of adjacent structures. Most lesions start out radiolucent, some progress to become mixed radiolucent and radiopaque, and others become homogenously radiopaque. The actual appearance of a lesion can vary with the stage of pathogenesis. A radiolucent lesion suggests that there has been lysis of normal bone. ${ }^{4}$ Radiolucent abnormalities within otherwise hard tissues result from decreased mineralization, decreased thickness, or a combination of the two, including normal, developmental, traumatic, inflammatory, cystic, neoplastic, osteodystrophy, metabolic/systemic, idiopathic, and foreign body artifacts. ${ }^{5}$ Despite the possible contribution of radiolucency to acute inflammation of the jaws and soft tissues, the relationship of acute pericoronitis and bone resorption has not taken into account the possible effects of aging.

The mandibular third molar has the highest risk of infection as frequently seen by acute inflammation in the elderly. The reason for removal of impacted lower third molars is pericoronitis or caries in $30 \%$ of patients and for prophylactic reasons 
in $31 \%$ of young people (mean age, 20 years) with impacted mandibular third molars ${ }^{6}$ extending any complications. Case reports of severe acute inflammation resulting from pericoronitis have shown necrotizing fasciitis. ${ }^{7,8}$ Similarly, most impacted mandibular third molars removal in the elderly are due to pericoronitis, and are a reminder of its association with coronal radiolucency. Furthermore, the diagnosis of radiolucency around the mandibular third molar is too ambiguous to understand the status of the molar, excluding unequivocal bone resorption by tumor or cyst, and most radiolucencies remain incompletely understood, including the effects of aging on radiolucency.

We hypothesized that the clinical pericoronal features of pericoronitis resulting from the absence of periodontium and cement of teeth simplify the identification of the radiographic findings of pericoronal radiolucency, which could be an indicator of acute inflammation. However, acute inflammation could be rare in patients without pericoronal radiolucency. We attempted to elucidate whether the underlying pericoronal radiolucency below the crown of the mandibular horizontal impacted third molar, which represents loss of periodontium, could be effective in showing acute inflammation, and whether substantial pericoronal radiolucency below the crown of the mandibular horizontal third molar could be associated with acute inflammation in aging. We suggest a relationship between acute pericoronitis, which could result from chronic inflammation, and pericoronal radiolucency below the crown with aging. Advances in the understanding of pericoronal bone resorption at sites where enamel contacts bone may offer insight into the complicated function of the periodontium. The relation between pericoronal bone resorption and acute inflammation associated with individuals older than 41 years will be discussed.

\section{Methods}

Patients were referred to Oral and Maxillofacial Surgery at the Matsumoto Dental University Hospital for a consultation or to treat the mandibular third molar between March 1989 and July 2007. Mesioangular, horizontal incompletely impacted third molars, in which the crown is partly at the level of the alveolar bone ridge, were monitored regarding pericoronal radiolucency below the crown of the tooth to the cementoenamel junction. A healthy finding without pericoronal radiolucency was defined by a uniform line without a rupture or a diffuse lucent area below the crown (Figure 1). Pericoronal radiolucency below the crown was defined by a diffuse lucent area due to irregular widening of a follicular space, ranging in width from 0 to $3.5 \mathrm{~mm}$

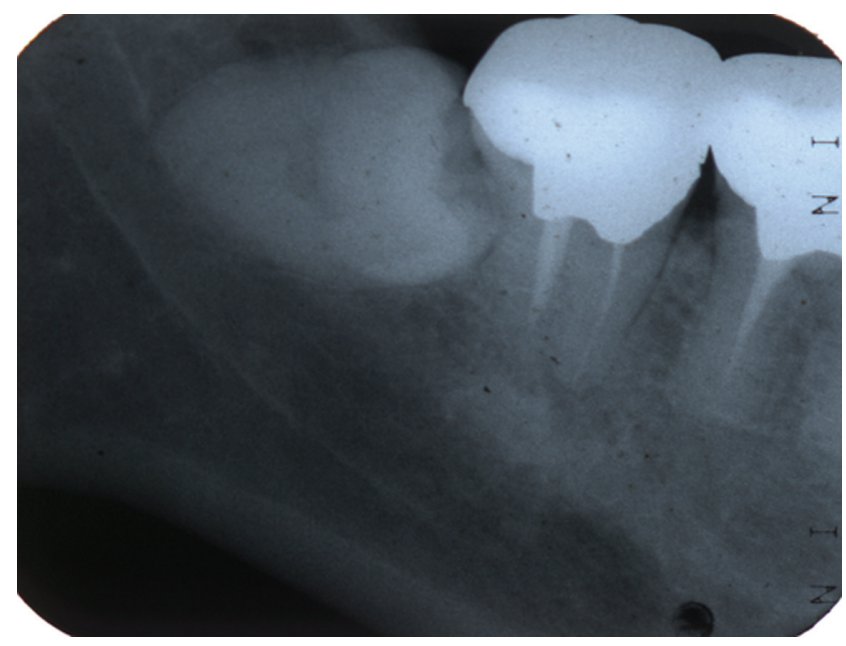

Figure I Radiograph of a 58-year-old woman with acute pericoronitis without pericoronal radiolucency below the crown.

in maximum amount below the crown (Figures 2, 3). The mean age \pm standard deviation (SD) of 200 men was $50.09 \pm 8.44$ (range, 41-74), and that of 146 women was $50.10 \pm 7.47$ (range, 41-72). No significant difference between them was shown $(P=0.99)$. The mean age $\pm \mathrm{SD}$ of 215 patients with pericoronal radiolucency below the crown was $50.29 \pm 8.35$ (range, 41-74) and the mean \pm SD of 131 patients without pericoronal radiolucency below the crown was $49.11 \pm 7.40$ (range, 41-70) (as shown in four patients with bilateral teeth). No significant difference was observed between the group of patients with and without pericoronal radiolucency $(P=0.18)$. Dental radiographs from 217 teeth of 215 patients with pericoronal radiolucency and 133 teeth of 131 patients without pericoronal radiolucency were assessed by an initial examination.

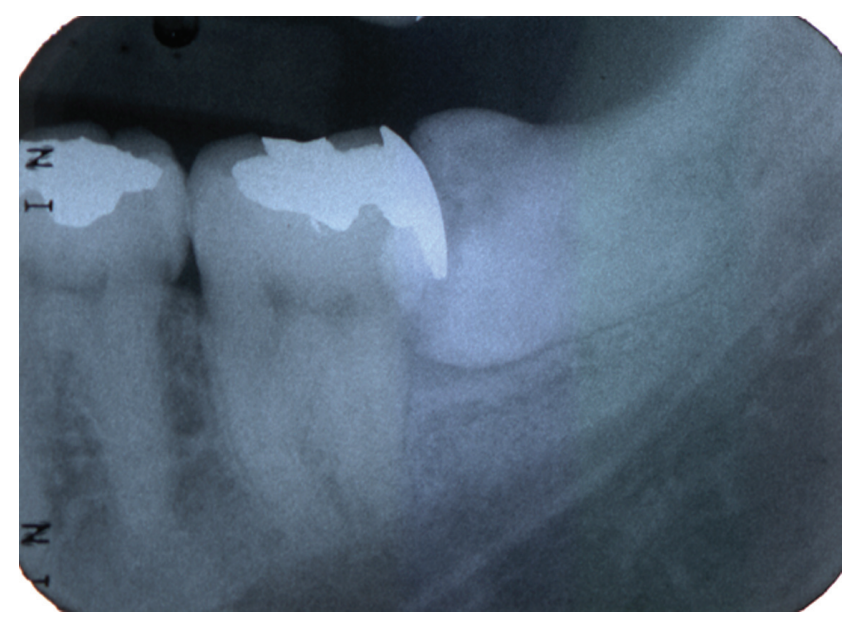

Figure 2 Radiograph of a 4 I-year-old man with acute pericoronitis with pericoronal radiolucency below the crown. 


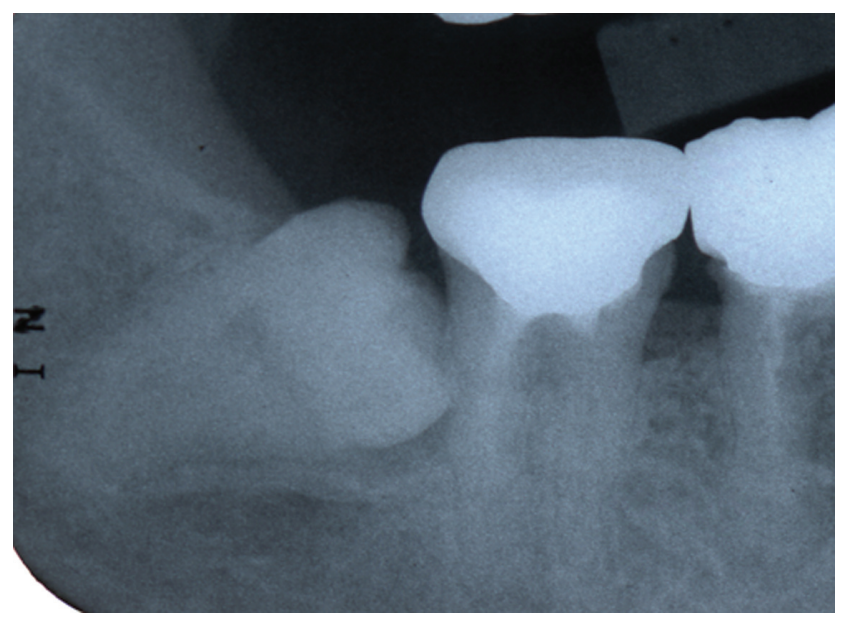

Figure 3 Radiograph of a 48-year-old woman with acute pericoronitis with pericoronal radiolucency below the crown.

Patients were classified based on the presence/absence of acute pericoronitis and those with or without pericoronal bone resorption below the crown of the horizontal incompletely impacted third molar. Acute pericoronitis showed redness, swelling, tenderness on palpation, and/or purulent gingival discharge with spontaneous pain of the gingival and alveolar mucosa in the region of the mandibular third molar. Severe acute inflammation was a complication which extended from the acute pericoronitis in the incompletely impacted third molar. Patients with the following conditions were excluded from those selected at the initial examination: cases of periodontitis of the mandibular second molar with radiolucency in the aspects of the mesial surface of the mesial root and the alveolar crest between the mesial and distal roots; apical radiolucency of the mandibular second or third molar; a completely impacted third molar; restoration ending below the cementoenamel junction of the second molar; the third molar stimulated mechanically by opposing teeth; cases radiographically diagnosed as tumor, keratcystic odontogenic tumor, dentigerous cyst; osseous lesion in the second and/or third molars; and HIV.

\section{Radiographic assessment}

Radiographs were obtained with an X-ray machine (Morita, Tokyo; Asahi, Kyoto, Japan) and Kodak Ultra-speed dental film (Eastman Kodak, Rochester, NY, USA) at $70 \mathrm{kVp}$ and $20 \mathrm{~mA}$ in 0.32 seconds or Kodak InSight dental film (Eastman Kodak) at $70 \mathrm{kVp}$ and $7 \mathrm{~mA}$ in 0.16 seconds using the parallel technique. Radiolucency of the pericoronal bone below the third molar was assessed by visual observation at $2 \times$ magnification. Bone resorption on radiographs was measured twice by the same experienced oral surgeon.
The second examination was one month after the initial examination. When the measurement of the presence or absence of bone resorption was different between the first and second times, the case was excluded from the study.

\section{Statistical analysis}

Mean values \pm SD of age among patient groups were calculated and analyzed using Student's $t$-test. The measurements were analyzed using $2 \times 2 \mathrm{t}$ Excel (Microsoft, Redmond, WA, USA) statistical software. The results of comparisons between the prevalence of acute inflammation in those with and without pericoronal radiolucency below the crown and between decades of age were assessed by chi-squared tests, and the influence of potentially confounding variables was further analyzed using logistic regression analysis. Significance was accepted at the $95 \%$ confidence interval $(\mathrm{CI})$ level $(P<0.05)$.

\section{Results}

In men older than 61 years, 13 teeth with acute inflammation $(46.3 \%)$ of 28 teeth were not more significant than 32 teeth with acute inflammation (26.0\%) of 123 teeth in men aged 41-50 years and 11 teeth with acute inflammation (22.4\%) of 49 teeth in men aged of 51-60 years (Table 1). In women older than 61 years, seven teeth with acute inflammation $(50.0 \%)$ of 14 teeth were more significant than 15 teeth $(16.1 \%)$ of 93 teeth in women aged $41-50$ years $(P<0.05)$ and five teeth with acute inflammation (11.6\%) of 43 teeth in women aged $51-60$ years $(P<0.05)$.

In the analysis between teeth with and without pericornal radiolucency, 58 teeth of 217 teeth with radiolucency and 25 teeth of 133 teeth without radiolucency showed acute pericoronitis (Table 1) with no significant difference in frequency between them. Acute inflammation was severe in six (2.8\%) of 217 patients with pericoronal radiolucency participating in this study, whereas there was no acute severe inflammation in patients without pericoronal radiolucency. Six patients were men with acute periostitis aged 42 years without history of disorder, 53 with diabetes mellitus, 68 with history of large intestine carcinoma, 71 with gastric ulcer and rheumatoid arthritis, and 74 with prostatauxe, and one man aged 61 years with a submandibular space abscess and diabetes mellitus. None of the women had a severe form of acute inflammation. Women aged more than 61 years with pericoronal radiolucency were more likely to develop acute inflammation compared to those aged $41-50$ years (odds ratio [(OR] ):9.77, 95\% CI: $1.67-57.29 ; P<0.05)$ and $51-60$ years (OR: 26.25, 95\% CI: 2.94-234.38; $P<0.01$ ). Men were at 
Table I Acute inflammation in teeth with or without radiolucency

\begin{tabular}{|c|c|c|c|c|c|c|c|c|c|}
\hline \multirow{2}{*}{$\begin{array}{l}\text { Age (y) } \\
4 I-50\end{array}$} & \multirow{2}{*}{$\begin{array}{l}\text { Gender } \\
\text { Men }\end{array}$} & \multirow{2}{*}{$\begin{array}{l}\text { With } \\
\text { radiolucency }\end{array}$} & \multicolumn{2}{|c|}{$\begin{array}{l}\text { Acute } \\
\text { inflammation }\end{array}$} & \multirow{2}{*}{$\begin{array}{l}\text { Severe acute } \\
\text { inflammation } \\
I^{c}\end{array}$} & \multirow{2}{*}{$\begin{array}{l}\text { Without } \\
\text { radiolucency }\end{array}$} & \multicolumn{2}{|c|}{ Acute inflammation } & \multirow{2}{*}{$\begin{array}{l}\text { Severe acute } \\
\text { inflammation }\end{array}$} \\
\hline & & & 20 & $26.32 \%$ & & & 12 & $25.53 \%$ & \\
\hline & Women & 54 & $1 \mathrm{I}^{\mathrm{a}}$ & $20.37 \%$ & 0 & 39 & 4 & $10.26 \%$ & 0 \\
\hline \multirow[t]{2}{*}{$5 I-60$} & Men & 35 & 9 & $25.71 \%$ & 1 & 14 & 2 & $14.29 \%$ & 0 \\
\hline & Women & 23 & $2^{\mathrm{b}}$ & $8.70 \%$ & 0 & 20 & 3 & $15.00 \%$ & 0 \\
\hline \multirow[t]{2}{*}{$\geq 61$} & Men & 22 & II & $50.00 \%$ & $4^{c}$ & 6 & 2 & $33.33 \%$ & 0 \\
\hline & Women & 7 & $5^{\mathrm{a}, \mathrm{b}}$ & $71.43 \%$ & 0 & 7 & 2 & $28.57 \%$ & 0 \\
\hline \multirow[t]{3}{*}{ Total } & Men & 133 & 40 & $30.08 \%$ & 6 & 67 & 16 & $23.88 \%$ & 0 \\
\hline & Women & 84 & 18 & $21.43 \%$ & 0 & 66 & 9 & $13.64 \%$ & 0 \\
\hline & $M+W$ & 217 & 58 & $26.73 \%$ & 6 & 133 & 25 & $18.80 \%$ & 0 \\
\hline
\end{tabular}

Notes: ${ }^{\mathrm{a} p}<0.05^{\mathrm{b}, \mathrm{c}} \mathrm{p}<0.01$.

greater risk of severe acute inflammation. Men aged more than 61 years with pericoronal radiolucency were more likely to develop severe acute inflammation than those aged 41-50 years (OR: 16.67, 95\% CI: 1.76-158.27; $P<0.01$ ). Thus, the presence of pericoronal radiolucency below the crown in patients aged more than 61 years was a significant predictor of acute inflammation.

\section{Discussion}

We have shown that acute pericoronitis was not likely to be more prevalent in patients with radiolucency than without; therefore, radiolucency did not appear to be associated with acute inflammation. However, the frequency of acute inflammation in women aged 61 or older was significantly more than that in women aged 60 or younger. Moreover, acute inflammation increased markedly in women aged more than 61 years with radiolucency. The OR for acute inflammation was markedly increased to 9.77 compared with women aged 41-50 years and 26.25 compared to women aged 51-60 years. In men, the severe form of acute inflammation was increased markedly to 16.67 compared to men aged $41-50$ years. These increases indicate an increased risk of acute inflammation in the elderly with pericoronal radiolucency. Thus, pericoronal radiolucency may be, in part, governed by aging, which is probably attributable to decreased bone density in the regulation of bone homeostasis. Age may be a risk factor related to acute inflammation in patients with pericoronal radiolucency below the crown, where not only loss of healthy bone, but also previous loss of periodontium below the crown in horizontal incompletely impacted third molars, may be essential for acute inflammation. Pericoronal radiolucency below the crown is associated with high risk for acute inflammation since a risk of acute pericoronitis exists in the space between the crown and the bone, and our results established a basis for the effects of aging on pericoronal radiolucency below the crown in horizontal incompletely impacted third molars, although individuals younger than 40 years were not studied.

However, the present study indicated that just $26.7 \%$ of patients with pericoronal radiolucency below the crown may be associated with a risk of later acute inflammation. The facts of the lower frequency of acute inflammation in the teeth with pericoronal radiolucency below the crown, and a similar frequency to that without pericoronal radiolucency suggest that it should be investigated whether pericoronal radiolucency below the crown is a preexisting and underlying risk factor predisposing acute inflammation, or whether pericoronal radiolucency below the crown is a causal factor of acute inflammation, since acute inflammation may arise even if there is no pericoronal radiolucency below the crown. Indeed, radiolucency is diagnosed after 10-12 days, ${ }^{9} 2-3$ weeks ${ }^{10}$ after initial symptom, and not until $2-4$ weeks ${ }^{11}$ by conventional radiographs. Nevertheless, observation of pericoronal radiolucency below the crown is still expected to predict acute inflammation because of the higher frequency of acute inflammation in the elderly with pericoronal radiolucency as shown in the present study. Moreover, patients without pericoronal radiolucency did not develop serious complications. This result suggests the possibility of previous acute inflammation in the patients with pericoronal radiolucency.

Severe acute inflammation was not seen in women, which indicates a possible gender-related difference, and suggests a differences in attitudes to health between men and women, and good oral hygiene care to control pericoronitis in women, although oral hygiene was not examined in the current study. This is supported by the better oral health-related lifestyle of women than men. ${ }^{12,13}$ 
Previously, an association was seen between pericoronal radiolucency below the crown and acute pericoronitis in diabetics, which is related to infection. ${ }^{14}$ This association indicates that acute inflammation does not act through a radiolucency-independent mechanism, but the factors of aging, male gender, or diabetes are essential to activate the chronic inflammation shown by pericoronal radiolucency, which is useful to predict acute inflammation when such factors are observed. We cannot exclude the possibility of the effect of systemic diseases on the regional conditions, since any systemic diseases may have altered the chronic inflammation or pericoronal radiolucency. However, we have no concern that systemic diseases would affect acute pericoronitis and/or pericoronal radiolucency more than diabetes mellitus. However, data on the past history of systemic diseases were not collected in our study, except in six cases who had shown severe acute inflammation. The frequency of acute inflammation might be different in differing diseases, but an increase in acute inflammation in the elderly might be related to a reduction of their resistance to acute inflammation. This suggests that association of systemic disease with infection would be important for prediction of acute inflammation, but many examples of various diseases which may effect infection are needed to draw solid conclusions.

Again, radiolucency includes various conditions. Some radiographically "normal" third molar impactions show a pathologically dentigerous cyst ${ }^{15}$ in third molar impactions in men aged 20-30 years old. ${ }^{16}$ In patients aged $13-24$ years, the dental follicle of third molars with incomplete root formation shows inactive enamel-reduced epithelium, inactive epitheium remnant, dense connective tissue, chronic inflammation, and calcification. ${ }^{17}$ These findings are a pathognomonic sign in young adults, and therefore may seldom occur in patients aged more than 41 years, excluding carcinomas around the third molar. We did not find such pathological lesions in patients who underwent tooth extraction in the current study.

Our findings may suggest, in part, the potential for early, although X-ray identification of patients at risk of acute inflammation is not be when compared to computed tomography "early" in comparison with CT or magnetic resonance imaging, identification of patients at increased risk for acute inflammation in the elderly, because pericoronal radiolucency could not be fully anticipated to improve in the elderly, whereas pericoronal radiolucency stabilizes in chronic conditions with worsened or improved stimuli in a young population who have few risk factors for acute inflammation. However, pericoronal radiolucency below crown may not always be related to inflammation, or may be in homeostasis based on "sustained" anti-inflammatory response. Therefore, factors leading to acute inflammation in radiolucency need to be studied, such as the regulation of the nuclear factor-kappa B (NF- $\mathrm{\kappa B})$ pathway is involved in many diseases associated with chronic inflammation in the immune response to infection. ${ }^{18-20}$ And, although the study design makes it impossible to determine cause and effect, these results may suggest that loss of regulatory control of acute or chronic inflammation caused by insufficient oral hygiene, phobia to dental treatment, or asymptomatic under chronic inflammation increase the prevalence of acute inflammation. These possibilities could lead to a change in care for the prevention of acute inflammation in the elderly. The effectiveness of early removal of third molar with pericoronal radiolucency may be shown for acute inflammation in the elderly. More analyses of the regional and/or systemic factors make it possible to achieve prognosis and specialized treatment in patients with incompletely impacted third molars, and may be important to prevent unnecessary treatment. These measurements emphasize the need for follow-up evaluation of the changes in susceptibility to acute inflammation and pericoronal radiolucency below the crown to enable therapeutic decision-making.

These results confirm the association between aging and acute inflammation, including severe inflammation accompanying pericoronal radiolucency below the crown of the mandibular horizontal, incompletely impacted third molar in patients aged more than 61 years.

\section{Acknowledgments}

We would like to thank Ms Shiho Kobayashi for her contribution to this study. The authors report no conflicts of interest in this work.

\section{References}

1. Haring JI, Lind LJ. Dental Radiography: Principles and techniques Philadelphia, PA: WB Saunders; 1996.

2. Frommer HH, Stabulas-Savage JJ. Radiology for the Dental Professional. 8th ed. St. Louis, MO: Elsevier Mosby; 2005.

3. Iannucci JM, Howerton LJ. Dental Radiography: Principles and techniques. 3rd ed. St. Louis, MO: Saunders Elsevier; 2006.

4. Goaz PW, White SC. Oral Radiology. 3rd ed. St. Louis, MO: Mosby; 1994.

5. Brocklebank L. Dental Radiology. Oxford, UK: Oxford University Press; 1997.

6. von Wowern N, Nielsen HO. The fate of impacted lower third molars after the age of 20. A four-year clinical follow-up. Int J Oral Maxillofac Surg. 1989;18(5):277-280.

7. Mizuno I, Mizutani H, Ueda M, Kaneda T. Temporal necrotizing infection of dental origin. J Oral Maxilloofac Surg. 1993;51(1):79-81. 
8. Ndukwe KC, Fatusi OA, Ugboko VI. Craniocervical necrotizing fasciitis in Ile-Ife, Nigeria. Br J Oral Maxillofac Surg. 2002;40(1):64-67.

9. Capitanio MA, Kirkpatrick JA. Early roentgen observations in acute osteomyelitis. Am J Roentgenol Radium Ther Nucl Med. 1970;108(3):488-496.

10. Davies HT, Carr RJ. Osteomyelitis of the mandible: complication of routine dental extractions in alcoholics. Br J Oral Maxillofac Surg. 1990;28(3):185-188.

11. Schuknecht BF, Carls FR, Valavanis A, Sailer HF. Mandibular osteomyelitis: evaluation and staging in 18 patients, using magnetic resonance imaging, computed tomography and conventional radiographs. J Craniomaxillofac Surg. 1997;25(1):24-33.

12. Murtomaa H, Metsäniitty $\mathrm{M}$. Trends in toothbrushing and utilization of dental services in Finland. Community Dent Oral Epidemiol. 1994;22(4):231-234.

13. Christensen LB, Petersen PE, Krustrup U, Kjøller M. Self-reported oral hygiene practices among adults in Denmark. Community Dent Health. 2003;20(4):229-235.

14. Yamaoka M, Ono Y, Ishizuka M, Yasuda K, Uematsu T, Furusawa K. Radiolucency below the crown of mandibular horizontal incompletely impacted third molars and acute inflammation in men with diabetes. Clinical, Cosmetic and Investigational Dentistry. 2009;1:27-34.
15. Glosser JW, Campbell JH. Pathologic change in soft tissues associated with radiographically 'normal' third molar impactions. $\mathrm{Br} \mathrm{J}$ Oral Maxillofac Surg. 1999;37(4):259-260.

16. Mesgarzadeh AH, Esmailzadeh H, Abdolrahimi M, Shahamfar M. Pathosis associated with radiographically normal follicular tissues in third molar impactions: a clinicopathological study. Indian J Dent Res. 2008;18(3):208-212.

17. de Oliveira DM, de Souza Andrade ES, de Silveira MM, Camargo IB. Correlation of the radiographic and morphological features of the dental follicle of third molars with incomplete root formation. Int J Med Sci. 2008;5(1):36-40.

18. Courtois G, Gilmore TD. Mutations in the NF-kappaB signaling pathway: implications for human disease. Oncogene. 2006;25(51): 6831-6843.

19. Ghosh S, Hayden MS. New regulators of NF-kappaB in inflammation. Nat Rev Immunol. 2008;8(11):837-848.

20. Hayden MS, Ghosh S. Shared principles in NF-kappaB signaling. Cell. 2008;132(3):344-362.
Clinical Interventions in Aging

\section{Publish your work in this journal}

Clinical Interventions in Aging is an international, peer-reviewed journal focusing on evidence-based reports on the value or lack thereof of treatments intended to prevent or delay the onset of maladaptive correlates of aging in human beings. This journal is indexed on PubMed Central, MedLine, the American Chemical Society's 'Chemical

\section{Dovepress}

Abstracts Service' (CAS), Scopus and the Elsevier Bibliographic databases. The manuscript management system is completely online and includes a very quick and fair peer-review system, which is al easy to use. Visit http://www.dovepress.com/testimonials.php to read real quotes from published authors. 